\title{
Risk Factors for Multi-drug Resistant Tuberculosis in Addis Ababa, Ethiopia
}

\author{
Fikadu Tadesse
}

Department of Health Studies, University of South Africa (UNISA), Ethiopia

Copyright $@ 2015$ Horizon Research Publishing All rights reserved.

\begin{abstract}
Multi-Drug resistant Tuberculosis (MDR-TB) is becoming a major challenge of the tuberculosis (TB) control program in Ethiopia. The objective of the study was to investigate risk factors for MDR-TB in Addis Ababa. Comparative cross-sectional study was conducted to determine the risk factors for MDR-TB in Addis Ababa. MDR and non MDR-TB cases were compared on key socio-demographic and patient related clinical features for multidrug-resistance. A total 439 (113 MDR-TB and 326 non MDR-TB) were enrolled in to the study. Standard data abstraction instrument were used. Data were entered and analysed using SPSS version 18 for windows. Initial descriptive analyses of all variables of interest, odds ratio, univariate and subsequent multiple regression analysis was carried out. Male sex (OR 2, 95\%CI 1.4-5), younger age of 15-34 years (OR 2.8, 95\%CI 1.7-6.4), twice or more episodes of TB treatment (OR 14, 95\%CI 1.3-9), treatment category of failure after re-treatment (OR 4, 95\%CI 2.5-6.83), pulmonary TB (OR 2, 95\%CI 1.7-5) and TB/HIV co-infection (OR 2.66, 95\%CI 1.32-5.6) were associated with the risks of becoming ill with MDR-TB. The national TB prevention and control programme should be given priority for routine Line Probe Assay to make early diagnosis of MDR-TB particularly among those identified risk groups.
\end{abstract}

Keywords Multi-drug Resistant Tuberculosis, MDR-TB, TB, Factors

\section{Introduction}

Tuberculosis (TB) remains a major global health problem. It causes ill-health among millions of people each year and ranks as the second leading cause of death from an infectious disease worldwide, after the Human Immunodeficiency Virus (HIV). The latest estimates includes there were 8.6 million new TB cases in 2012 and 1.3 million TB deaths. Globally, the absolute number of TB cases is increasing slowly, although the number of cases per capita is falling by around $1 \%$ per year $[1,2]$.
Global control of TB has been jeopardized by two major threats: HIV and Acquired Immuno-Deficiency Syndrome (AIDS) and Multi-Drug Resistant Tuberculosis (MDR-TB) [2]. MDR-TB is defined as strains of Mycobacterium tuberculosis that are resistant to at least isoniazid and rifampicin. Drug-resistant TB threatens global TB control and is a major public health concern in several countries. In 2012, data from drug resistance surveys and continuous surveillance among notified TB cases suggest that $3.6 \%$ of newly diagnosed TB cases and $20 \%$ of those previously treated for TB had MDR-TB worldwide [1].

TB is one of the leading infectious diseases in Ethiopia. The country ranks $7^{\text {th }}$ in the list of the world's 22 high burden countries for TB with incidence estimated at 379/100,000 for all forms of TB and 168/100,000 for smear positive TB. The healthcare delivery system in Ethiopia emphasises primary healthcare, including preventive, promotive and basic curative services which involves prevention and control of diseases, including TB $[3,4]$.

Drug resistant TB has also become a major challenge of the TB control program in Ethiopia. WHO 2008 TB report estimates 2,400 new MDR-TB cases occur every year in Ethiopia [4,5]. In a study conducted by 2010 in Addis Ababa, MDR-TB was observed in one of the 44 new cases $(2.3 \%)$ and $45 / 63$ previously treated patients. Drug susceptibility testing against second-line drugs (SLDs) showed that $26.1 \%$ of all MDR-TB isolates were susceptible to all SLDs tested and $73.9 \%$ were resistant to one or more classes of SLDs $(71.4 \%)$. The same study indicated T3_ETH was the predominant spoligotype, followed by CAS_KILI [6]. In this regard, the Ethiopian Government has identified MDR-TB as one of priority public health problems and initiated comprehensive treatment for MDR-TB cases. One such measure includes endorsement of single procurement mechanisms for TB drugs and controlled use of second line anti-TB drugs since 2009. Furthermore TB operational research is to be strengthened within the overall health system aligned to the national health policy and health sector development program [3].

Treatment of MDR-TB is more challenging. It requires use of second-line drugs that are more costly and cause more severe side-effects, and recommended regimens must be 
taken for up to two years $[7,8]$. The MDR-TB treatment strategy in Ethiopia combines standardised and individualized treatment based on second line culture and Drug Susceptibility Testing (DST) (kanamycin and Ofloxacin) in all confirmed MDR-TB patients. Standardised regimens have given to all confirmed MDR-TB cases under daily Directly Observed Therapy (DOT). The initial phase is at least six months, and then the continuation phase is at least 12 months. In Ethiopia, the standard regimen for MDR-TB uses the combinations of: [Ethambutol- Pyrazinamide Kanamycin (Amikacin)- Levofloxacin - Ethionamide Cycloserine] for six months, and [Ethambutol Pyrazinamide - Levofloxacin - Ethionamide - Cycloserine] for 12 months. The total duration may be extended by clinicians according the findings of culture conversion [4].

Like most TB control programmes, the National Treatment Program does not have the resources to perform culture and Drug and Sensitivity Test (DST) for all TB patients [4]. More over the study area is characterized by high population growth, density and massive migration of peoples from regions. As a result, the proportion and risk of MDR-TB infection is estimated to be high. Despite the rising public health concern of MDR-TB in the country, factors underlying for spread of this diseases is not determined. Thus, this study is believed to fulfil the existing information gap and help in designing appropriate prevention and control strategies. Therefore the aim of the study was to determine risk factors for MDR-TB in Addis Ababa, Ethiopia.

\section{Materials and Methods}

\subsection{Study Setting and Period}

The study was undertaken at 26 public health centers in Addis Ababa city from 1 January 2008 to December 30, 2011. Addis Ababa is the capital city of Ethiopia. It is the largest city in Ethiopia, with a population of 3,384,569 according to the 2007 population census [9].

\subsection{Study Design}

A comparative cross-sectional study was conducted to determine the risk factors for MDR-TB in Addis Ababa, Ethiopia. MDR-TB and non MDR-TB patients were compared on key socio-demographic and patient related clinical features for drug resistance.

\subsection{Study Population}

The study population comprised all MDR and non MDR-TB cases who were registered for TB treatment follow up in 26 public health centres in Addis Ababa from 1January 2008 to December 30, 2011. During the study period TB treatment were provided at 26 public health centres in the city of Addis Ababa. MDR-TB cases were those patients diagnosed and confirmed using culture and DST while non MDR-TB cases were those either AFB, clinical or radiological evidence of sensitive tuberculosis for whom complete medical records were available for inclusion. MDR and non MDR-TB cases where medical records are incomplete were excluded from the study.

\subsection{Sample Size Determination}

A Fleiss sample size calculation formula using Epi Info version 3.5.1 for windows were used to calculate the sample size. The sample size was determined based on proportion of exposure of MDR-TB and non MDR-TB among previous TB treatment. According to the Federal Ministry of Health of Ethiopia (FMOH), MDR-TB among re-treatment cases is $12 \%$ compared to $2 \%$ among primary TB patients [4]. A marginal error of 3\%, $80 \%$ study power, $95 \%$ confidence limit and a ratio of 1:3 MDR to non MDR were assumed to enhance precision of the study. Consequently, the sample size was determined as follows:

$$
\mathrm{n}_{1}=\frac{\left[\mathrm{Z}_{\frac{\alpha}{2}} \sqrt{(r+1) P Q}+\mathrm{Z}_{1-\beta} \sqrt{\left(r p_{1} q_{1}+p_{2} q_{2}\right)}\right]^{2}}{r\left(p_{1}-p_{2}\right)^{2}},{ }_{2}={ }_{1}
$$

$\mathrm{n}_{1}$-number of MDR-TB cases, $\mathrm{n}_{2}$-number of non MDR-TB cases, $\mathrm{Z} \alpha$-standard score corresponding $95 \%$ of certainty (1.96), $\mathrm{Z}_{1-\beta}^{\overline{2}}$-is power of the study $(80 \%)$, $\mathrm{p}_{1}$-proportion of MDR-TB among previously treated (12\%), and $\mathrm{p}_{2}$-proportion of MDR-TB among new cases $(2 \%)$, r-ratio of MDR to non MDR-TB (1:3), $\mathrm{q}_{1}=1-\mathrm{p}_{1}, \mathrm{q}_{2}=1-\mathrm{p}_{2}, \mathrm{P}=\frac{p_{1}+r p_{2}}{r+1}$, $\mathrm{Q}=1-\mathrm{P}$. Accordingly, a total of 400 study participants ${ }^{r+1}(100$ MDR-TB and 300 non MDR-TB) were included in this study. $10 \%$ of the samples were added to cater for incomplete data making the total sample size of 439 (113 MDR-TB and 326 non MDR-TB).

\subsection{Sampling Procedure}

Both MDR and non MDR-TB cases were selected using systematic random sampling at each health centres. The number of non MDR-TB cases from each site was allocated proportionally to the load of MDR-TB cases contributed at the site. For systematic random sampling list of existing MDR-TB and non MDR-TB at each health centre was used as a sampling frame. By calculating the sample interval, the first TB patient was selected using lottery method and subsequent patients were selected by adding the calculated sampling interval for each health centres.

\subsection{Data Collection Method and Quality Assurance}

The data collection instruments were designed for a standard record review format containing study variables. Secondary data was obtained from the registry of national TB control program, patient cards, and charts. The forms had the following information: patient ID, age, sex, location of residence, history of previous TB treatment, episodes of TB treatment, treatment category, site of involvement and HIV 
status. Data abstraction instrument were pretested before the actual data collection were took place. Data collectors were TB focal nurses who are familiar with national unit TB register. Records that meet eligibility criteria based on culture, DST, clinical and laboratory results confirmed diagnosis were selected. Consultation with medical officers and nurses providing medical service for TB patients were done to measure the construct domain of the study to ensure instruments validity.

\subsection{Data Entry and Analysis}

All data from the questionnaire were coded and entered into the Statistical Packages for Social Science (SPSS) version 18 (Chicago, Illinois), checked and explored for data integrity. Initial descriptive analyses of all variables of interest such as frequency and percentages were employed to analyse the results. Odds ratios were calculated to determine the strength of association between selected variables. Univariate analysis was carried out in order to identify potential risk factors associated with drug resistance in TB treatment and subsequently, multiple regression analysis was carried out with the set of variables that showed strong association in the univariate model. Statistical inference was made at $P$ value $<0.05$.

\subsection{Ethical Consideration}

The study was approved by the University of South Africa postgraduate research and ethics committee and Addis Ababa City Administration Health Bureau (AACAHB). Based on ethical clearance a request for permission letter has detailed explanation of the research itself, the reasons for the research and kind of research to be conducted in the facility were provided to the authorities at the study sites by AACAHB. All explicit patient identifiers were removed to ensure anonymity of abstracted medical data while maintaining the integrity of the medical records. No personal identifiers of the study participants, such as name and address were included in the data abstraction form.

\section{Results}

\subsection{Demographic Data}

Medical records were reviewed for 113 and 326 patients with MDR and non MDR-TB respectively, diagnosed between 1 January 2008 to December 30, 2011. Of the total, $239(54 \%)$ were males and $200(46 \%)$ were females. Among the MDR, $73(64.6 \%)$ were males and $40(35.4 \%)$ were females; while of the non MDR 166 (51\%) were males and $160(49 \%)$ were females. The youngest TB subject was 5 years old, while the oldest was 77 years old. The mean age was 29 years (standard deviation of 14). More than $60 \%$ of both MDR and non-MDR patients were 15-34 years old. 362 $(82.2 \%)$ were urban residents whereas $78(17.8 \%)$ came from rural areas. Urban residents made up 103 (91\%) of the MDR-TB patients and 259 (79\%) among non MDR-TB patients.

\subsection{Clinical Features}

Among the MDR-TB cases, 112 (97\%) have previously been treated for TB in comparison to $31(10 \%)$ of the non MDR-TB. 295 (90.5\%) of the non MDR-TB had no history of previous TB treatment compared to $1(1 \%)$ of the MDR-TB cases. Of all MDR-TB cases, 90 (90\%) were treated twice or more times for tuberculosis in comparison to $25(8 \%)$ of the non MDR groups. Failure after re-treatment constitutes $72(64 \%)$ among MDR-TB cases and $6(2 \%)$ for non MDR. 100 (88.5\%) and 216 (66\%) of the MDR and non MDR were respectively infected with pulmonary TB. Voluntarily HIV counselling and testing is performed routinely for all TB patients as a result all patients were tested for HIV during the treatment period. Almost half, 54 (48\%) of MDR-TB and 85 (26\%) of the non MDR cases were HIV positive.

\subsection{Multiple Logistic Regression Analysis}

Demographic variables, age and sex were found to be associated with MDR in univariate analysis. Similarly clinical features such as previous history TB treatment, twice or more times history of TB treatment, treatment category of failure after re-treatment, pulmonary involvement of TB and TB/HIV co-infection were found to be associated with MDR-TB in univariate analysis. After multivariate analysis, aside from geographical location of residence, which just failed to reach statistical significance (OR 1.6, 95\%CI $0.5-5, P=0.396$ ), other potential risk factors were associated with MDR-TB.

\section{Discussion}

The emergence and spread of multi-drug resistant tuberculosis MDR-TB is threatening to destabilize global tuberculosis control. Ethiopia is among 27 high MDR-TB burden countries that carry $87 \%$ of the total global burden and one of the four countries in Africa (i.e. South Africa, Nigeria, and DRC)[3,4]. It was with this fact that the study was done in order to determine contributing factors for emerging public health problems of MDR-TB. The study investigated different factors such as socio-demographic and clinical features in order to determine risk factors for MDR-TB. 
Table 1. Socio-demographic characteristics and clinical features of MDR and non MDR: Factors in the Multivariate analysis

\begin{tabular}{|c|c|c|c|c|c|c|}
\hline Characteristics & $\begin{array}{c}\text { MDR-TB } \\
(\mathrm{n}=113)\end{array}$ & $\begin{array}{c}\text { Non MDR-TB } \\
(\mathrm{n}=326)\end{array}$ & $\begin{array}{l}\text { Crude OR } \\
(95 \% \mathrm{CI})\end{array}$ & p-value & $\begin{array}{c}\text { Adjusted } \mathrm{OR}^{\mathrm{a}} \\
(95 \% \mathrm{CI})\end{array}$ & p-value \\
\hline $\begin{array}{c}\text { Sex: } \\
\text { Male } \\
\text { Female }\end{array}$ & $\begin{array}{l}73 \\
40 \\
\end{array}$ & $\begin{array}{l}166 \\
160\end{array}$ & $\begin{array}{c}1.76(3-7.7) \\
1.00\end{array}$ & 0.012 & $\begin{array}{c}2(1.4-5) \\
1.00\end{array}$ & 0.039 \\
\hline $\begin{array}{c}\text { Geographical location of reside } \\
\text { Urban } \\
\text { Rural } \\
\end{array}$ & $\begin{array}{c}103 \\
10 \\
\end{array}$ & $\begin{array}{c}258 \\
68 \\
\end{array}$ & $\begin{array}{c}2.7(1.35-5.5) \\
1.00 \\
\end{array}$ & 0.005 & $\begin{array}{c}1.6(0.5-15) \\
1.00 \\
\end{array}$ & 0.396 \\
\hline $\begin{array}{c}\text { Age (in yrs.): } \\
<14 \\
15-34 \\
35-54 \\
+55 \\
\end{array}$ & $\begin{array}{c}2 \\
72 \\
32 \\
7\end{array}$ & $\begin{array}{c}15 \\
199 \\
83 \\
29\end{array}$ & $\begin{array}{c}1.11(0.7-1.2) \\
12(4.8-19) \\
1.8(0.9-7) \\
1.00 \\
\end{array}$ & $\begin{array}{l}0.487 \\
0.001 \\
0.221\end{array}$ & $\begin{array}{c}0.02(0.51-1.02) \\
2.8(1.7-6.4) \\
1.4(0.08-8) \\
1.00 \\
\end{array}$ & $\begin{array}{c}0.981 \\
0.004 \\
0.91\end{array}$ \\
\hline $\begin{array}{c}\text { Previous history of TB treatme } \\
\text { Yes } \\
\text { No }\end{array}$ & $\begin{array}{c}112 \\
1\end{array}$ & $\begin{array}{c}11 \\
295\end{array}$ & $\begin{array}{c}1.6(38-148) \\
1.00\end{array}$ & 0.00 & $\begin{array}{c}11(5-9.67) \\
1.00\end{array}$ & 0.00 \\
\hline $\begin{array}{c}\text { Episode of TB treatment: } \\
\text { Once } \\
\geq 2 \text { times } \\
\text { Not used before } \\
\end{array}$ & $\begin{array}{c}22 \\
90 \\
1 \\
\end{array}$ & $\begin{array}{c}6 \\
25 \\
295 \\
\end{array}$ & $\begin{array}{c}1.00 \\
10(3.8-27) \\
1.00 \\
\end{array}$ & 0.00 & $\begin{array}{c}1.00 \\
14(1.3-9) \\
1.00 \\
\end{array}$ & 0.024 \\
\hline $\begin{array}{c}\text { Category of TB treatment: } \\
\text { Failure of first treatment } \\
\text { Relapse } \\
\text { Return after default } \\
\text { Failure after re-treatment } \\
\text { New (Primarily acquired) }\end{array}$ & $\begin{array}{c}19 \\
10 \\
11 \\
72 \\
1 \\
\end{array}$ & $\begin{array}{c}6 \\
15 \\
4 \\
6 \\
295 \\
\end{array}$ & $\begin{array}{c}3.17(0.36-27) \\
0.4(0.05-3.5) \\
0.7(4.56-44) \\
6(2-15) \\
1.00 \\
\end{array}$ & $\begin{array}{c}0.297 \\
0.409 \\
0.031 \\
0.0011\end{array}$ & $\begin{array}{c}0.29(0.91-7) \\
0.23(0.15-8.1) \\
6(.1-10) \\
4(2.5-6.83) \\
1.00 \\
\end{array}$ & $\begin{array}{c}0.741 \\
0.260 \\
0.29 \\
0.003\end{array}$ \\
\hline $\begin{array}{c}\text { Site of TB involvement: } \\
\text { Pulmonary } \\
\text { Extra-pulmonary } \\
\end{array}$ & $\begin{array}{c}100 \\
13\end{array}$ & $\begin{array}{l}216 \\
100 \\
\end{array}$ & $\begin{array}{c}3.2(3.54-10.7) \\
1.00\end{array}$ & 0.002 & $\begin{array}{c}2(1.7-5) \\
1.00\end{array}$ & 0.00 \\
\hline $\begin{array}{l}\text { HIV test result: } \\
\text { Positive } \\
\text { Negative }\end{array}$ & $\begin{array}{l}59 \\
54 \\
\end{array}$ & $\begin{array}{c}5 \\
241 \\
\end{array}$ & $\begin{array}{c}1.7(7-24) \\
1.00 \\
\end{array}$ & 0.00 & $\begin{array}{c}2.66(1.32-5.6) \\
1.00 \\
\end{array}$ & 0.012 \\
\hline
\end{tabular}

aThe data were analysed using univariate and multivariate logistic regression.

Each odds ratio has adjusted for all other characteristics in the table.

OR-odds ratio, aOR-adjusted odds ratio, CI-confidence interval, $\mathrm{p}<0.05$

The study showed being male is a risk factor for becoming ill with MDR-TB. Similarly, studies has revealed male sex was associated with the risk of becoming ill with MDR-TB $[2,10,11]$. The higher prevalence of MDR-TB in men over women may be explained by the fact that women are more compliant with treatment and therefore less likely to receive inadequate treatment than men. Furthermore, men are almost always outdoors and therefore more susceptible to community-acquired resistant strains [12].

More than $60 \%$ proportion of both MDR and non-MDR TB patients were among young adults aged 15-34 years old $[2,13,14]$. Age-related differences in treatment adherence are also possible, as younger patients are often occupied by study, work or other activities on a daily basis, in contrast with the more sedentary lifestyle post-retirement.

Evidences showed previous history of anti-tuberculosis treatment has been found to pose the greatest risk for the development of MDR-TB [2,15,16,17]. Patients who received previous anti-tuberculosis treatment had a higher fold increased odds of acquiring multidrug resistance and prior exposure to anti-TB drugs is a well-established risk factor for drug resistance. Among those previously treated TB patients, twice or more times history of TB treatment were at highest risk of developing MDR-TB in comparison to those who were treated less frequently. Frequent treatment may arise due to high chances of defaulting of TB patients from treatment. Studies in Pakistan showed, $80 \%$ of the MDR-TB patients used anti-TB treatment course twice or more times [18].

TB treatment category was associated with occurrence of MDR-TB. Patients who fail after re-treated for TB were higher fold riskier in developing MDR-TB relative to other treatment categories. Strong association of MDR-TB were demonstrated in those fails after re-treated [19]. This suggests a need for clear medication guide and provision constant reminders for patients during their DOTs to prevent acquisition of MDR-TB. The national TB control programme doesn't have the capacity to perform DST for all TB patients and for all MDR suspects [5]. Thus, priority should be given for routine DST for those failed after previously re-treated to make early diagnosis of MDR-TB. Furthermore, the TB control programme could have to consider Line Probe Assay (LPA) for rapid diagnosis of MDR suspects over conventional DST as it can perform high volume of a test per day and facilitate early initiation of correct treatment and appropriate measures to prevent transmission [20].

Based on these findings, it is plausible to assume that 
pulmonary involvement of TB is associated more with MDR-TB. Similarly, the study conducted in Hong Kong, China and Tomsk, Russian Federation showed vast majority of MDR-TB cases suffered from pulmonary TB [13,21].

In this study, strong association was demonstrated between TB/HIV co-infection and the risk of becoming ill with MDR-TB. HIV infected patients are 1.7 times more likely to acquire MDR-TB. The slightly increased risk of MDR-TB among TB/HIV co-infected individuals reiterates the findings of other studies $[22,23,24]$. TB infected patients must be frequently screened for HIV and vice versa. In order to minimize the risk of MDR-TB, Anti-Retroviral therapy (ART) should be initiated for all TB/HIV co-infected patients as soon as possible. A special attention must be given to selection of appropriate TB and ART treatment regimens in order to avoid the risk of increased drug reaction and resistance. Furthermore, early diagnosis of MDR-TB using LPA has to give priority for those TB/HIV co-infected individuals.

Finally, the implication of the study findings is clear that the important information about the common risk factors for occurrence of MDR-TB which emerged from this research should be incorporated in the National TB Prevention and Control programme. This information would have critical benefit in TB patient education, improvement in quality of TB care and prevention of MDR-TB.

This study has some limitations. Firstly it is limited by its cross-sectional design and use of secondary data for analysis. A further limitation is that these findings cannot be directly generalised to all regions in the county other than Addis Ababa because of socio-economical, epidemiological and health system related variations in TB and MDR-TB occurrences.

\section{Conclusions}

It was found to be male sex, younger age, twice or more episodes of TB treatment, TB treatment category of failure after re-treatment, pulmonary involvement and HIV infection were the identified risk factors for becoming ill with MDR-TB. As the treatment for MDR-TB is often difficult, the TB prevention and control strategy could have to consider rapid diagnosis using LPA with more emphasis to those identified susceptible groups. The prevention of this lethal public health problem of MDR-TB requires combined effort of all stake holders, namely the government, the health care workers, patient and community.

\section{Acknowledgements}

My heartfelt thanks go to the authorities at health facilities, Sub-City Health Offices of Addis Ababa and health care workers who were working in TB clinics. I want to thank Addis Ababa City Administration Health Bureau and all UNISA staffs for their support.

\section{REFERENCES}

[1] World Health Organization, Global tuberculosis control: WHO report 2013, WHO, Geneva, Switzerland, 2013

[2] World Health Organization, Multidrug and extensively drug-resistant TB (M/XDR-TB):Global report on surveillance and response, WHO, Geneva, Switzerland, 2010

[3] Federal Ministry Health of Ethiopia TB Research Advisory Committee, Roadmap for Tuberculosis Operational Research in Ethiopia. FMOH, Addis Ababa, Ethiopia, 2013

[4] Federal Ministry Health of Ethiopia, Guideline for program and clinical management of drug resistant Tuberculosis. FMOH, Addis Ababa, Ethiopia, 2009

[5] World Health Organization, Global tuberculosis control: surveillance, planning, financing: A WORLD FREE OF TB 2009. WHO, Geneva, Switzerland, 2009

[6] M. Agonafir, E. Lemma, D. Wolde-Meskel, S. Goshu, A. Santhanam, F. Girmachew, D. Demissie, M. Getahun, M. Gebeyehu, D. van Soolingen. Phenotypic and genotypic analysis of multidrug-resistant tuberculosis in Ethiopia, INT J TUBERC LUNG DIS,vol.14, pp.1259-1265, 2010

[7] World Health Organization Towards universal access to diagnosis and treatment of multidrug-resistant and extensively drug-resistant tuberculosis by 2015 . WHO, Geneva, Switzerland, 2011.

[8] Marahatta, SB. Multi-drug resistant tuberculosis burden and risk factors: An update, Kathmandu University Medical Journal, Vol. 8, no.1, pp.116-125, 2010

[9] Central Statistical Authority of Ethiopia (CSA), The 2007 population and housing census result of Ethiopia, UNFPA, Addis Ababa, Ethiopia, 2008

[10] Molly F. Franke, Sasha C. Appleton, Jaime Bayona, Fernando Arteaga, Eda Palacios, Karim Llaro, Sonya S. Shin, Mercedes C. Becerra, Megan B. Murray, and Carole D. Mitnick. Risk Factors and Mortality Associated with Default from Multidrug-Resistant Tuberculosis Treatment, Clin Infect Dis, vol.46, pp.1844-1851, 2012

[11] A Faustini, A J Hall, C A Perucci. Risk factors for multidrug resistant tuberculosis in Europe: a systematic review, Thorax, vol. 61, pp.158-163, 2006

[12] Muayad A. Merza, Parissa Farnia, Payam Tabarsi, Mehdi Khazampour, Mohammad Reza Masjedi, Ali Akbar Velayati. Anti-tuberculosis drug resistance and associated risk factors in a tertiary level TB centre in Iran: a retrospective analysis, J Infect Dev Ctries, vol. 5, no.7, pp.511-519, 2011

[13] S. Law, W. W. Yew, C. Chiu Leung, K. M. Kam, C. M. Tam, C. K. Chan, C. C. Leung. Risk factors for multidrug-resistant tuberculosis in Hong Kong, INT J TUBERC LUNG DIS, vol.12, no.9, pp.1065-1070, 2008

[14] Waseem Saeed, Arshad Naseem, Jamal Ahmed J Ayub. Retrospective audit of patients treated for MDR-TB in re-treatment category, Med Coll Abbottabad, vol. 21, no.2, pp.94:8, 2009

[15] Kliiman, K. Highly drug resistant tuberculosis in Estonia: 
Risk factors and predictors of poor treatment outcome. Estonia, Tartu University press, 64, 2009

[16] Kai Kliiman, Alan Altraja. Predictors of Extensively Drug-Resistant Pulmonary Tuberculosis, Ann Intern Med, vol.150, pp.766-775, 2009

[17] Alistair D. Calver, Alecia A. Falmer, Megan Murray, Odelia J. Strauss, Elizabeth M. Streicher,Madelene Hanekom, Thelma Liversage, Mothusi Masibi, Paul D. van Helden, Robin M. Warren, and Thomas C. Victor. Emergence of Increased Resistance and Extensively Drug-Resistant Tuberculosis Despite Treatment Adherence, South Africa, Emerging Infectious Diseases $\bullet$ CDC, vol.16, pp.2, 2010

[18] Fazli Wahab, Saadia Ashraf, Naveed Khan, Rukhsana Anwar and Mukhtiar Zaman Afridi. Risk Factors for Multi-Drug Resistant Tuberculosis in Patients at Tertiary Care Hospital, Peshawar, Journal of the College of Physicians and Surgeons Pakistan, volume 19, no. 3, pp.162-164, 2009

[19] Desiree TB D'souza, Nerges F Mistry, Tina S Vira, Yatin Dholakia, Sven Hoffner,Geoffrey Pasvol, Mark Nicol and Robert J Wilkinson. High levels of multidrug resistant tuberculosis in new and treatment-failure patients from the Revised National Tuberculosis Control Programme in an urban metropolis (Mumbai) in Western India, BMC Public Health, vol.9, pp.211, 2009

[20] Yadav RN, Singh BK, Sharma SK, Sharma R, Soneja M, Sreenivas V, Myneedu VP, Hanif M, Kumar A, Sachdeva KS, Paramasivan CN, Vollepore B, Thakur R, Raizada
N, Arora SK, Sinha S. Comparative evaluation of GenoType MTBDRplus line probe assay with solid culture method in early diagnosis of multidrug resistant tuberculosis (MDR-TB) at a tertiary care centre in India, PLoS One, Vol.5, no.8, pp.9, 2013

[21] IY Gelmanova, S Keshavje, VT Golubchikova, VI Berezina, AK Strelis, GV Yanova, S Atwoodd \& M Murray.et al. Barriers to successful tuberculosis treatment in Tomsk, Russian Federation: non-adherence, default and the acquisition of multidrug resistance, Bulletin of the World Health Organization, vol.85, pp.9, 2007

[22] Neel R. Gandhi, N. Sarita Shah, Jason R. Andrews, Venanzio Vella, Anthony P. Moll, Michelle Scott, Darren Weissman, Claudio Marra, Umesh G. Lalloo, and Gerald H. Friedland. HIV Co-infection in Multidrug- and Extensively Drug-Resistant Tuberculosis Results in High Early Mortality, Am J Respir Crit Care Med, vol.181, pp. 80-86, 2010

[23] Suchindran S, Brouwer ES, Van Rie A. Is HIV Infection a Risk Factor for Multi-Drug Resistant Tuberculosis? A Systematic Review, PLoS ONE, vol. 4, pp. 5, 2009

[24] Scott K Heysell, Tania A Thomas, Neel R Gandhi, Anthony P Moll, François J Eksteen, Yacoob Coovadia, Lynette Roux, Palav Babaria, Umesh Lalloo,Gerald Friedland and Sarita Shah. Blood cultures for the diagnosis of multidrug-resistant and extensively drug-resistant tuberculosis among HIV-infected patients from rural South Africa: a cross-sectional study, BMC Infectious Diseases, vol. 10, pp. 344,2010 